\title{
Case Report of New Onset of Adult Diabetes due to Obesity with Socioeconomic barrier
}

\author{
Adel El Naggar* \\ Department of Pathology, The University of Texas, MD Anderson Cancer Center, USA
}

Submission: October 22, 2016; Published: October 27, 2016

*Corresponding author: Adel El Naggar, Department of Pathology, The University of Texas, MD Anderson Cancer Center, Houston, Texas, USA, Email: anaggar@mdanderson.org

\section{Abstract}

Introduction: Diabetes Mellitus (DM); defined as a Group of Diseases (Cardio-metabolic Disorders/Complex Metabolic Disorders) Characterized by Hyperglycemia Due To an Absolute or Relative Lack of Insulin or to a Cellular Resistance to Insulin. Diabetes had multiple health hazards \& high incidence of cardio metabolic comorbidities due to the nature of disease progress, leading to diabetic complications \& disabilities which impair individual productivity towards the community. Prevalence of adult diabetes worldwide is growing forcibly to affect the adolescence especially in gulf areas \& Americas due to unhealthy life styles\& physical inactivity. The most common cause of adult diabetes is the Obesity. Diabetes \& obesity are the highest prevalence of the gulf area; Saudi Arabia considered highest real country worldwide in diabetes \& obesity prevalence.

Case Report: I present a case of a 20 year-old adult Saudi (white) male, obese physically in-active without past medical history or any significant habit of medical importance; with new Onset of adult Diabetes due to Obesity with Socioeconomic barrier. Patient had family history of DM/hypertension/ischemic heart disease (IHD)/Dyslipidemia.Came to my endocrine/diabetes clinic at July 2015; Cash patient (not covered by insurance); financial barrier; with classical symptoms of diabetes: Polyuria, Tiredness, Weight loss, Thirst sensation, blurring of vision. His weight $105 \mathrm{~kg}$, height $177 \mathrm{~cm}$, calculated body mass index (BMI) was $33.5 \%$, his blood pressure $130 / 70 \mathrm{mmHg}$, his systematic physical \& clinical examination was normal; however he was symptomatic, his random blood sugar (RBS) test was elevated; $430 \mathrm{mg} / \mathrm{dl}$.

Laboratory work up screening tests confirmed the diagnosis of newly discovered adult diabetes done for baseline new diabetes assessment, positive results were; fasting plasma blood sugar (FBS) $246 \mathrm{~g} / \mathrm{dl}$; postprandial plasma blood sugar (PPBS) $412 \mathrm{mg} / \mathrm{dl}$; glycated hemoglobin test (HbA1c) 10.8\%. Case managed as an obese newly diagnosed adult type 2 DM; managed by early use of basal insulin analogue (Detemir) for reversal of gluco-toxicity symptomatic illness \& resuming anabolism (basal insulin gradually withdrawn over first two months ) plus Metformin/ Sitagliptin combinations oral anti-diabetic agent (due to socio-economic impact); concerning weight reduction \& improve insulin sensitivity \& resting/preserve pancreatic Beta-cell function. The highly positive effect with Weight reduction encouragement regimen \& Positive role of psychological support lead to progressive improvement \& control of patient's Diabetes \& Obesity; although socio-economic impact.

Discussion: This patient's case of adult type 2 DM with Obesity is common seen in worldwide; especially gulf (middle east) \& Americas. Suchlike cases are missed by routine management of blood sugar level apart from the focus on the main cause of their diabetes, which is the obesity in adults. Control of diabetes not only means blood sugar control but also should be causality control. Role of weight reduction is very important here towards causality control for patient's diabetes, furthermore multiple cardio metabolic risks outcomes associated with both diabetes \& obesity; aim of lowering insulin resistance by encourage weight loss is beneficial although unhealthy life style in gulf area \& patient's socio-economic status whichaffecting drug choices ideally preferred in such cases like injectable Glucagon-like peptide-1 (GLP-1) Analogues; liraglutide (high cost) \& Sodium-glucose co-transporter 2 (SGLT2) inhibitors which not yet launched in Saudi Arabia. Due to these challenges; I chose first basal insulin Detemir plus metformin-sitagliptin oral anti-diabetic combination as regard weight concern/cost/glucotoxicity control/ preserve pancreatic Beta cell function; then maintenance on metformin-sitagliptin combination plus weight reduction motivation by good doctor-patient relationship \& play on good psychological support. Specify the target aim for patient's control of his diabetes by weight loss regimen which by regular follow-up leads to achieving our target for both controlling patient's diabetes \& obesity.

Keywords: Adult Diabetes; Obesity; Cardio metabolic risks outcomes; Diabetes causality control; metformin-sitagliptin combination; Glucotoxicity; Beta cell function; Weight reduction concern; Socio-economic impact; Psychological support.

Abbreviations: DM: Diabetes Mellitus; IHD: Ischemic heart disease; BMI: Body mass index; RBS: Random blood sugar; FBS: Fasting plasma blood sugar; PPBS: Postprandial plasma blood sugar; HbA1c: Glycated hemoglobin; GLP-1: Glucagon-like peptide-1; SGLT2: Sodium-glucose cotransporter 


\section{Introduction}

Diabetes is a group of chronic diseases characterized by hyperglycemia [1]. Diabetes had multiple health hazards \& high incidence of cardio metabolic co morbidities due to the nature of disease progress, leading to diabetic complications \& disabilities which impair individual productivity towards the community. Modern medical care uses a vast array of lifestyle and pharmaceutical interventions aimed at preventing and controlling hyperglycemia. In addition to ensuring the adequate delivery of glucose to the tissues of the body, treatment of diabetes attempts to decrease the likelihood that the tissues of the body are harmed by hyperglycemia. Micro-vascular and Macro-vascular Complications of Diabetes [2]. Prevalence of adult diabetes worldwide is growing forcibly to affect the adolescence especially in gulf areas \& Americas due to unhealthy life styles, physical inactivity, environmental \& genetic factors.Saudi Arabia according to international diabetes federation 2013; first real country worldwide in adult diabetes prevalencemore than $24 \%$ of the population. The most common cause of adult diabetes is the Obesity [3].

Overweight and obesity are defined as abnormal or excessive fat accumulation that may impair health. BMI is a simple index of weight-for-height that is commonly used to classify overweight and obesity in adults. It is defined as a person's weight in kilograms divided by the square of his height in meters $\left(\mathrm{kg} / \mathrm{m}^{2}\right)$. The fundamental cause of obesity and overweight is an energy imbalance between calories consumed and calories expended. Globally, there has been: an increased intake of energy-dense foods that are high in fat; and an increase in physical inactivity due to the increasingly sedentary nature of many forms of work, changing modes of transportation, and increasing urbanization. Raised BMI is a major risk factor for non-communicable diseases such as: serious diet-related chronic diseases, including type 2 diabetes, cardiovascular disease, hypertension and stroke, and certain forms of cancer. Overweight and obesity are the fifth leading risk for global deaths.

At least 2.8 million adults die each year as a result of being overweight or obese. Worldwide obesity has more than doubled since 1980. In 2014, more than 1.9 billion adults, 18 years and older, were overweight. Of these over 600 million were obese. $39 \%$ of adults aged 18 years and over were overweight in 2014, and $13 \%$ were obese [3]. Most of the world's population lives in countries where overweight and obesity kills more people than underweight. Obesity is preventable. Diabetes \& obesity are the highest prevalence of the gulf area; Saudi Arabia considered highest real country worldwide in both diabetes \& obesity prevalence [4].

\section{Case Report}

In my endocrine/diabetes clinic; I am facing a large scale variety of patients; which mainly diabetic patients whether with/ without complications \& with/without co-morbidities. I present a case of a 20 year-old adult Saudi (white) male, obese physically in-active without past medical history or any significant habit of medical importance; with new Onset of adult Diabetes due to Obesity with Socioeconomic barrier. Patient had family history of DM/hypertension/ischemic heart disease (IHD)/Dyslipidemia. Patient came to my endocrine/diabetes clinic; Cash patient (not covered by insurance); financial barrier; with classical symptoms of newly onset adult diabetes: Polyuria, Tiredness, Weight loss, Thirst sensation, blurring of vision. His weight 105 $\mathrm{kg}$, height $177 \mathrm{~cm}$, calculated body mass index (BMI) was 33.5\%, his blood pressure $130 / 70 \mathrm{mmHg}$, His systematic physical \& clinical examination was normal; however, he was symptomatic, His random blood sugar (RBS) test was elevated; 430mg/dl. Laboratory work up screening tests confirmed the diagnosis of newly discovered adult diabetes done for baseline new diabetes assessment, positive elevated laboratory results were; fasting plasma blood sugar (FBS) 246mg/dl; postprandial plasma blood sugar (PPBS) 412mg/dl; glycated hemoglobin test (HbA1c) $10.8 \%$; serum cholesterol $247 \mathrm{mg} / \mathrm{dl}$; serum TG $201 \mathrm{mg} / \mathrm{dl}$.

LDL-cholesterol $175 \mathrm{mg} / \mathrm{dl}$, while other laboratory tests were normal KFTs/LFTs/CBC, also done insulin level was $22.1 \mathrm{~m}$ unit/ $\mathrm{mL}$ (normal value 2.6-24.9) \& C-peptide level was $2.9 \mathrm{ng} / \mathrm{mL}$ (normal value 0.9-7.1) \& ABG was no acidosis; $\mathrm{PH}$ was 7.337; $\mathrm{HCO} 3$ $26.6 \mathrm{mmol} / \mathrm{L}$.Case managed as an obese newly diagnosed adult type 2 DMAs regard his \& symptoms/CV risks/low socioeconomic status; plan of management aim to fight both patients problems; obesity \& control his new onset symptomatic diabetes, by early use of basal insulin analogue (Detemir; which has less effect on weight gain among basal insulin analogues) for reversal of glucotoxicity symptomatic illness \& resuming anabolism : starting dose was 20IU subcutaneous injection once daily (insulin Detemir; gradually withdrawn over first two months ) plus Sitagliptin/ Metformin 50/1000mg combinations oral anti-diabetic agent twice daily (due to socio-economic impact which let option of GLP-1 analogue difficult as regard cost \& SGLT2 inhibitors not used as regard availability in Saudi Arabia; not launched yet. Both GLP-1 analogue \& SGLT2 inhibitors was the first ideal choice for diabetic obese patients added on metformin therapy); concerning weight reduction \& improve insulin sensitivity \& resting/preserve pancreatic Beta-cell function from exhaustion \& failure.

Added on base of life style modification management for both obesity \& diabetes via exercise/diabetic low fat diet control/ weight reduction motivation/ good psychological support. Patient within one week restore his glycemic control PPBS was 145mg/ $\mathrm{dl}$; less symptomatic, after two months of gradual withdrawal of insulin Detemir; patient restore completely his diabetic control \& anabolism with no symptoms\& respond well to weight reduction regimen; which help in diabetes control, his FBS was average $96 \mathrm{mg} / \mathrm{dl}$ without insulin, patient was only on life style modification management plusSitagliptin/Metformin 50/1000 mg combinations oral twice daily. After 3 months from overall 
management plan, his $\mathrm{HbA1c}$ was 5.9\% (excellent controlled) with mean average blood sugar $123 \mathrm{mg} / \mathrm{dl}$. After another 3 months, with life style modification management plus Sitagliptin/ Metformin 50/1000mg combinations oral twice daily; patient was asymptomatic, healthy, active, clinically almost better.

His recent clinical data; weight $88 \mathrm{~kg}$ vs. $105 \mathrm{~kg}$; BMI about $28.2 \%$ vs. $33.5 \%$; blood pressure $118 / 67 \mathrm{mmHg}$, His systematic physical \& clinical examinations are normal; RBS 89mg/ dl. Laboratory work up follow up tests for assessment of the management plan, FBS $81 \mathrm{mg} / \mathrm{dl}$; PPBS $114 \mathrm{mg} / \mathrm{dl}$; HbA1c 5.6\% vs. $10.8 \%$ (excellent diabetes control); serum cholesterol 156mg/ dl; serum TG 146mg/dl; LDL-cholesterol 99mg/dl, with normal KFTs/LFTs. Now patient on Sitagliptin/Metformin 50/850mg combinations oral twice daily (Dose reduced) His CBS excellent controlled: His FBS not exceeds $110 \mathrm{mg} / \mathrm{dl}$ His PPBS not exceeds $140 \mathrm{mg} / \mathrm{dl}$ plus Life style modification, Exercise \& Psychological support; patient motivated for his weight reduction \& his DM control.

The highly positive effect with Weight reduction encouragement regimen \& Positive role of psychological support lead to progressive improvement \& control of patient's Diabetes \& Obesity; although socio-economic impact.

\section{Discussion}

Diabetes is a group of chronic diseases characterized by hyperglycemia [1]. Diabetes had multiple health hazards \& high incidence of cardio metabolic comorbidities due to the nature of disease progress, leading to diabetic complications \& disabilities which impair individual productivity towards the community. People with diabetes have an increased risk of developing a number of serious health problems. Consistently high blood glucose levels can lead to serious diseases affecting the heart and blood vessels, eyes, kidneys, nerves and teeth. In addition, people with diabetes also have a higher risk of developing infections. In almost all high-income countries, diabetes is a leading cause of cardiovascular disease, blindness, kidney failure, and lower limb amputation [5].

During the past three decades, the worldwide prevalence of diabetes has doubled [6,7]. A recent estimate predicts the number of people with diabetes will grow from 382 million adults in 2013 to 592 million by 2035 [7]. More than two-thirds of this increase (69\%) will occur in developing countries. Obesity is a major public health problem has been recognized for more than 40 years [8]. Obesity continues to increase particularly rapidly in those who are more overweight [9]. Obesity is an important contributor to the rising tide of diabetes. Although genetic factors provide a key background for these events and may account for up to $20 \%$ of the variance in $\mathrm{BMI}$, it is clear that environmental factors play a major role [10]. The global epidemics of type 2 diabetes and obesity have been attributed to the interaction between lifestyle changes and genetic predisposition to these diseases [3].
Cardio-metabolic health is a measure of your risk for diabetes and heart disease. It is determined by a set of conditions that Include weight, blood glucose levels and cholesterol. The worldwide prevalence of overweight and obesity is high and is increasing [11,12]. WHO estimates that more than 1.3 billion adults worldwide are overweight [3]. Cardio-metabolic diseases (cardiovascular disease, diabetes, and kidney disease) are leading causes of death in high-income countries (WHO, 2008). An increased risk of cardio-metabolic disease is associated with a lower socioeconomic status (SES) and ethnicity [13]. Insulin resistance refers to the state of decreased insulin response and is a common feature of obesity, hypertension, diabetes, and coronary artery disease [14]. Endothelial and insulin signaling pathways crosstalk each other and therefore the relationship between endothelial function and insulin metabolism is very important in disorders, such as hypertension, obesity, or diabetes.

Insulin resistance, a hallmark of metabolic syndrome, impairs vascular response and increases cardiovascular risk [14]. This patient's case of adult type 2 DM with Obesity is common seen in worldwide; especially gulf (middle east) \& Americas. Suchlike cases are missed by routine management of blood sugar level apart from the focus on the main cause of their diabetes, which is the obesity in adults. Control of diabetes not only means blood sugar control but also should be causality control. Role of weight reduction is very important here towards causality control for patient's diabetes, furthermore multiple cardio metabolic risks outcomes associated with both diabetes \& obesity [15]; aim of lowering insulin resistance by encourage weight loss is beneficial although unhealthy life style in gulf area \& patient's socio-economic status which affecting drug choices ideally preferred in such cases like injectable Glucagon-like peptide-1 (GLP-1) Analogues; liraglutide (high cost) \& Sodium-glucose co-transporter 2 (SGLT2) inhibitors which not yet launched in Saudi Arabia.

Due to these challenges; I chose first basal insulin Detemir plus metformin-sitagliptin oral anti-diabetic combination as regard weight concern/cost/glucotoxicity control/preserve pancreatic Beta cell function; then maintenance on sitagliptin/metformin combination plus weight reduction motivation by good doctorpatient relationship \& play on good psychological support. Specify the target aim for patient's control of his diabetes by weight loss regimen which by regular follow-up leads to achieving our target for both controlling patient's diabetes \& obesity.

*My vision in management of adult (type 2) diabetes, put these points in individualization of patient management decision;

- Weight concern in management (avoid weight gain medications in obese patients); add weight neutral/ reduction regimens for obese patient.

- Beta cell preservation regimen to avoid beta cell exhaustion/failure.

- Safety profile as regard high risk of hypoglycemia. 
- $\quad$ Patient overall profile as regard co morbid diseases \& KFTs/LFTs.

- Cardio metabolic risk reduction from outcomes of diabetes \& obesity.

- Individualization of each patient according to his lifestyle/occupation/level of education/socioeconomic/ age group etc. Added to overall patient profile (Figure 1-5).
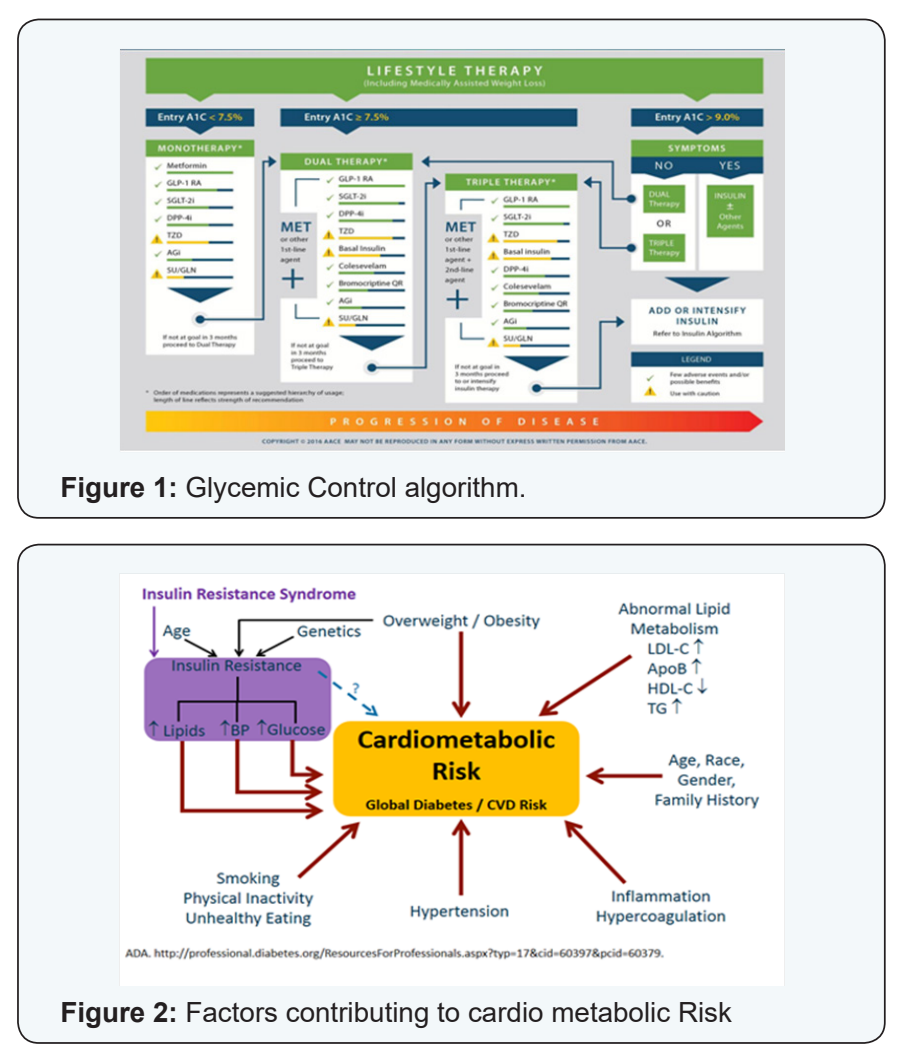

\section{Benefits of weight neutral/reduction regimens in type 2 diabetes}

\section{Just $1 \mathrm{~kg}$ body weight loss is associated with:}

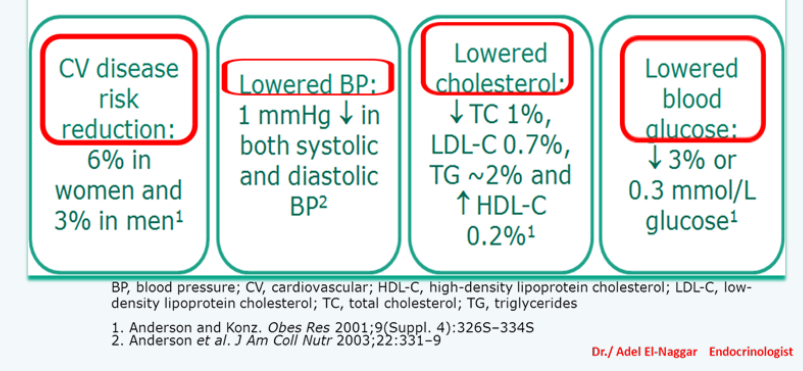

Figure 3: Benefits of weight neutral/reduction regimnes in type 2 diabetes.
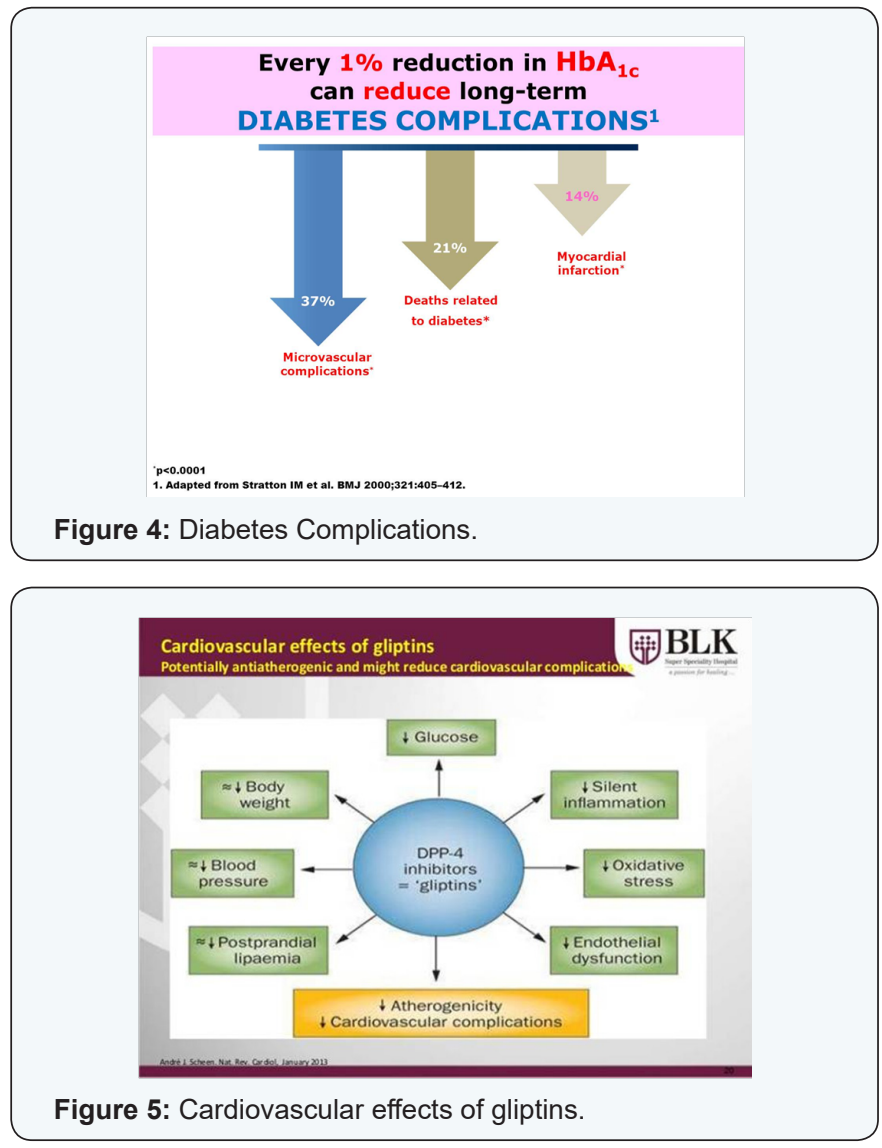

\section{References}

1. American Diabetes Association (2009) Diagnosis and classification of diabetes mellitus. Diabetes Care 32(Suppl. 1): S62-S67.

2. Fowler MJ (2008) Microvascular and macrovascular complications of diabetes. Clinical Diabetes 2008 Apr; 26(2): 77-82.

3. http://www.who.int/mediacentre/factsheets/fs311/en/

4. http://easo.org/education-portal/obesity-facts-figures/

5. http://www.idf.org/complications-diabetes

6. Whiting DR, Guariguata L, Weil C, Shaw J (2011) IDF diabetes atlas: global estimates of the prevalence of diabetes for 2011 and 2030. Diabetes Res Clin Pract 94(3): 311-321.

7. Guariguata L, Whiting DR, Hambleton I, Beagley J, Linnenkamp U, et al. (2014) Global estimates of diabetes prevalence for 2013 and projections for 2035. Diabetes Res Clin Pract 103(2): 137-149.

8. Bray GA (1976) Obesity in Perspective. Vol. 2, parts 1 and 2, Fogarty International Center Series on Preventive Medicine, DHEW Publication, Washington, DC, USA, pp. 75-708.

9. Sturm R, Hattori A (2013) Morbid obesity rates continue to rise rapidly in the United States. Int J Obes 37(6): 889-891.

10. Locke AE, Kahali B, Berndt SI, Justice AE, Pers TH, et al. (2015) Genetic studies of body mass index yield new insights for obesity biology. Nature 518(7538): 197-206.

11. Ng M, Fleming T, Robinson M, Thomson B, Graetz N, et al. (2014) Global, regional, and national Prevalence of overweight and obesity in 
children and adults during 1980-2013: a systematic analysis for the Global Burden of Disease Study 2013. Lancet 384(9945): s766-781.

12. NCD Risk Factor Collaboration (NCD-RisC) (2016). Trends in adult body-mass index in 200 countries from 1975 to 2014: a pooled analysis of 1698 population-based measurement studies with 19.2 million participants. Lancet 387(10026): 1377-1396.

13. Mackenbach JP, Stirbu I, Roskam AJ, Schaap MM, Menvielle G, et al (2008) Socioeconomic inequalities in health in 22 European countries. N Engl J Med 358(23): 2468-2481.
14. Rask-Madsen C, Kahn CR (2012) Tissue-specific insulin signaling, metabolic syndrome, and cardiovascular disease. Arterioscler Thromb Vasc Biol 32(9): 2052-2059.

15. Bray GA (2015) Diabetes and obesity-time bombs to be defused Diabetes Care 38(11): 1997-1999.

Your next submission with JuniperPublishers will reach you the below assets

- Quality Editorial service

- Swift Peer Review

- Reprints availability

- E-prints Service

- Manuscript Podcast for convenient understanding

- Global attainment for your research

- Manuscript accessibility in different formats ( Pdf, E-pub, Full Text, Audio)

- Unceasing customer service

Track the below URL for one-step submission http://juniperpublishers.com/online-submission.php 